\title{
Research on Gas-Insulated Switchgear flashover fault location system
}

\author{
XIE Shuang ${ }^{1, a}$, ZHU Jialin ${ }^{2, b}$, ZHU Shilong ${ }^{2, b}$ \\ ${ }^{1}$ Beijing information science and technology university, Beijing 100192, China \\ ${ }^{2}$ Beijing information science and technology university, Beijing 100192, China \\ ${ }^{3}$ Beijing information science and technology university, Beijing 100192, China \\ aemail: 271233309@qq.com, bemail:jlzhu@bistu.edu.cn, cemali:zhushilong8023@163.com
}

Keywords: GIS; Flashover fault location; Three op amp differential circuit; Wavelet denoising; ZigBee protocol

\begin{abstract}
This paper introduces the detection principle of Gas-Insulated Switchgear(GIS) ultrasonic testing method for the fault location of flashover.We designed the GIS flashover fault location monitoring system, which solved the problem of low precision and time-consuming for the detection of GIS.We designed the hardware and software of the monitoring system. We use the three op amp differential amplifier circuit in front processing circuit of the hardware part to suppress the common mode signal in the signals. When the signal is processed by software, the method of wavelet soft and hard threshold is designed to remove the noise in the signal and improve the signal to noise ratio.We achieve the task of wireless data transmission through the ZigBee2007Pro protocol stack.Experimental platform is built to verify the reliability of the GIS flashover fault location system, which can improve the positioning accuracy and fault location, and has the advantages of low cost, portability and so on.
\end{abstract}

\section{Introduction}

Gas-Insulated Switchgear (GIS) [6] improves the security of Ultra high voltage transmission in electric power system.Besides,GIS has the advantages of compact structure, small occupied space, fully enclosed type switch, high operating reliability, long service cycle, and so on [1-3].Recent years, the application of GIS in electric power system becomes more and more wide. But because the internal structure of GIS is complex and highly airtight [4], it is difficult to locate the fault location of GIS, which is complex and the cost is high.Meanwhile,GIS will result in a small number of internal defects due to production, assembly, transport and other various reasons.These small insulation defects can lead to GIS insulation failure, reduce the service life, and even cause flashover failure, which affects the operation of the power grid [4-5]. So,when flashover occurs, we must quickly locate the fault location, in order to reduce the harm to the safety of the people's property timely.

In the industrial field,the commonly used methods of fault location are ultra high frequency method, pulse current method, optical detection method, ultrasonic method and gas analysis method.In these methods,more widely used and more mature is the ultrasonic method.

\section{The principle and location method of GIS ultrasonic method to detect the flashover}

When the GIS flashover, accompanied by a high temperature, gas molecules will produce violent movements in the conditions of high temperature.The gas in the flashover position will produce vibration to form sound wave, and receive the acoustic signals through acoustic sensors, so as to realize the location of the flashover fault [8-9]. The propagation process of sound signal caused by flashover failure is more complicated in GIS interior.The attenuation of the sound signal is more serious,perhaps the attenuation amplitude can reach more than 10 times, when it arrived flange composed of epoxy resin and other materials [7]. Thus, we can install a sensor to capture the vibration signal strength in each section of the GIS .Due to the attenuation characteristics of the acoustic signal, the sensor installed in the GIS is different from the installation position, the 
intensity of the received acoustic signal is not the same, which can realize the location of the flashover.

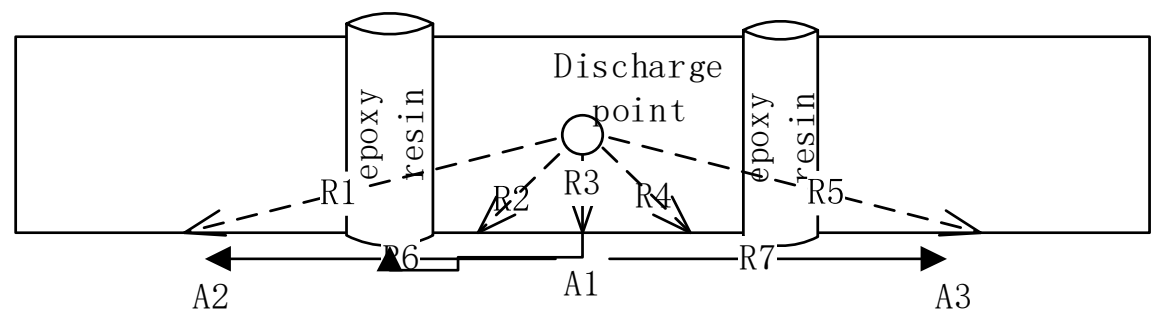

Figure 1. The transmission schemes of typical GIS flashover fault signal

As shown in Figure 1, assuming that the location of flashover fault is located in the center of the GIS gas chamber, then the sound signal generated by the flashover fault will be propagated along the path R1 to the path R5.It can rely on the sound signal monitored by strength detection node which is installed on the front end of the GIS to realize the fast positioning of flashover fault.

\section{Design of hardware system}

The whole system is mainly composed of the sensor, the hardware processing circuit, the wireless transmission platform and the host computer software platform. The Ultrasonic method is used to quickly locate the GIS internal flashover discharge, which is very easy to cause the error because of the noise source. Therefore, the effective extraction of the flashover signal is the key to the system design.

The Acoustic signal piezoelectric ceramic sensors are respectively arranged in each section of GIS equipment.When the signal is received by the sensor, the analog signal is converted to digital signal by $A / D$ conversion.Because the received signal contains a lot of white noise and noise, we need to adopt three op amp differential amplifier for preprocessing to suppress common mode signal effect. Secondly, the pre processed signal is amplified by a reverse proportion circuit; finally, a four order band-pass circuit is used to filter the amplified signal;In order to facilitate the processing of the back end of the microcontroller, we need to post amplification of the filter circuit. Singlechip is used by the STM32F3. The signal transmitted by the single chip microcomputer is transmitted to the host computer software through the wireless transmission protocol ZigBee. The schematic diagram of the structure is shown in Figure 2.1.

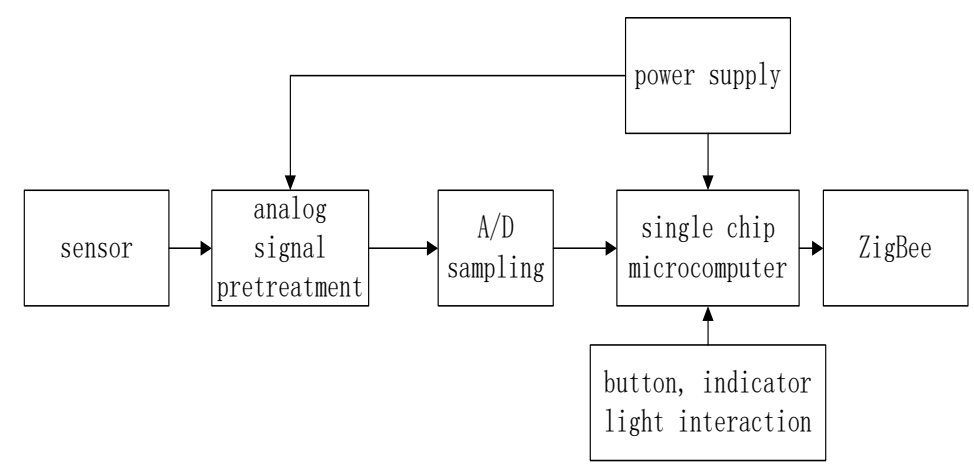

Figure 2.1 The structure diagram of a single detection device

\section{Design of software system}

System software program portion includes: A/D sampling process programming based on STM32F3 microcontroller, wireless data transmission programming based on ZigBee2007Pro and PC Monitoring Software Interface programming based on LabView. Where in the first portion realize the signal sampling process, to complete the anti-interference and filter software design of the analog signal. 
The keys of the wavelet analysis algorithm are the choice of threshold, signal decomposition and reconstruction. Because in the actual environment, the signal is very weak, so we use the amplifier circuit to 10 times the signal amplification, and then extract the signal.We use the combination of the single exponential decay and the double exponential decay model as the attenuation model.

Threshold selection plays an important role in signal reconstruction, and it is related to the degree of signal recovery.In the selection of threshold, we selected the heursure rules.In the threshold processing method, we use the soft and hard threshold processing method, the function expression is as follows:

$$
f_{h}(t)=\left\{\begin{array}{cc}
\operatorname{sign}(x(t))(|x(t)|-\lambda a) & |x(t)|>a \\
0 & |x(t)| \leq a
\end{array}\right.
$$

We carried out a series of simulation experiments, using Matlab simulation data to determine the correlation coefficient[11]. Correlation coefficient can reflect the similarity of the two signals, the greater the correlation coefficient, the higher the degree of similarity, the better the treatment.We determine the wavelet basis function and decomposition level according to the ratio of correlation coefficient.It is found that when the wavelet function is coif wavelet, the vanishing matrix is 5 ,the decomposition level is 6 , the signal correlation is the largest, that is, the signal reconstruction is the best.

\section{Experimental program}

Because the GIS flashover fault is a destructive fault, we have carried out a number of simulation experiments in the course of the experiment.There are one main type of experiment: the simulation experiment in the laboratory for GIS air chamber.

Experiment scheme:We use 3mm adhesive tape to stick sensor in the middle of the wall of the gas chamber, and then hit the wall of the same position to simulate the location of the flashover failure, using the speaker to replace the noise, and constantly moving the speaker's position.Finally, we use wavelet analysis to compare the data before and after using wavelet analysis, and then through the analysis of data to observe the effect of denoising effect.As is shown in Figure 3.1.

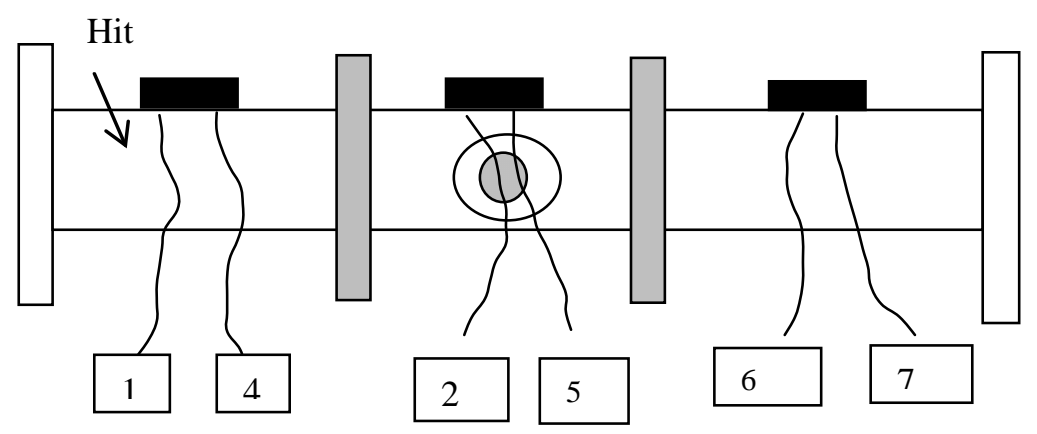

Figure 3.1 Schematic diagram of experimental program percussion

\section{Test result and analysis}

First, we need to determine the location of the tap, in a quiet environment, we use the device without the addition of wavelet algorithm (that is, 1,2,3 device) for the two experiments, recording data as a comparative benchmark,as is shown in Table 3.1.After the addition of noise the experimental data more in line with the rules of this group of data or closer to this group of data, the better the effect of denoising.Then, according to the Figure 3.1, put the speaker in a successive position (each test is only one position on the speaker), and then we hit two times in each position, the final two sets of test data were recorded. In order to ensure the consistency of each impact signal, we use an ejection device, so that each time the wall of the tube is basically similar to the attack. As is shown in Table 3.2. 
Table 3.1 Experiment data without noise

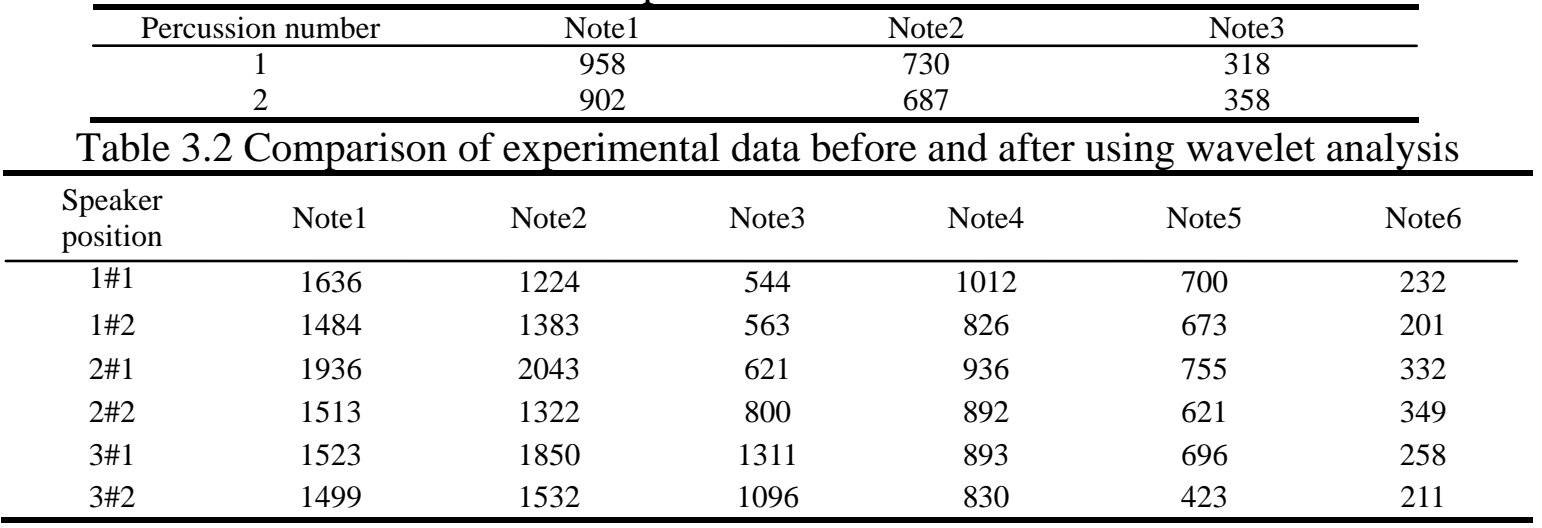

So, in the absence of wavelet analysis, with the noise position away from the tap position, the accuracy of detection is reduced such as $2 \# 1 、 3 \# 1 、 3 \# 2,2$ node data is the largest, but the actual tap position in the 1 node.However, after using the wavelet analysis, the scheme basically meets the attenuation law, and there is no the 2 node data larger than the 1 node data, and the accuracy of the program is higher.It shows that this method has a certain effect.

By comparing the experimental data , it can be concluded that the scheme has a better denoising effect,and the scheme improves the accuracy of the positioning system, in the strong noise environment, the accuracy rate of the system increased about $20 \%$.

\section{Conclusion}

Through the experimental verification of this scheme, it is concluded that the design can effectively remove the noise in the signal, and it can realize the fast location of the fault point and improve the accuracy of the location.And through the ZigBee wireless communication technology, the collected sound signal data is transmitted to the monitoring end of PC. But this circuit, there are still insufficient. The detection accuracy is reduced due to electromagnetic interference and other noise, so it should be further strengthened hardware filter circuit performance in the industrial field.The signal strength at the end of the GIS is larger than the signal at the end of the flashover fault when the flashover fault occurs in some particular locations.Preliminary analysis, due to the end of the metal seal with a signal caused by the echo, after superposition of signal strength appears “inflated".These problems need to be improved in the following work.

\section{References}

[1] Xuechen Luo. $S F_{6}$ Gas-Insulated Switchgear(GIS)[M]. Beijing:China Electric Power Press 1999:86-88.

[2] CHANG C, CHANG C S, JIN J, et al. Source classification of partial discharge for gas insulated substation using waveshape pattern recognition[J]. IEEE Transactions on Dielectrics and Electrical Insulation, 2005, 12(2): 374-386.

[3] Xin Li. Research on GIS partial discharge detection technology[D]. Beijing: North China Electric Power University. 2005.

[4] Zhangjian Wu,Chengrong Li, Bo Qi and so on. Comparison of the sensitivity of the ultra high frequency method and ultrasonic wave method in the partial discharge detection of GIS[J]. Modern electric power 2010, 27(3): 31-36.

[5]Chen Fuan, Tang Huizeng, Li Honggang. Application of On-Line Ultrasonic And UHF Partial Discharge Detection In 1000kV GIS[C].//Applied Mechanics and Materials Vols Transaction Technical Publications, 2014. 
[6] Lan Chu. The advantages of GIS equipment and its application[J].Meishan science and technology. 2005, 04:13 15+47

[7] Bihong Guo. Research on fault location and internal discharge monitoring of GIS flashover[J].Huatong Technology,1997,01:15 18

[8] Fei Zhao. Partial discharge detection technology for gas insulated fully enclosed device[J]. Rural electrification,2008,04:17 18.

[9] Md, Enamul, Haque, Ahmad, Bin, etc. Application of Acoustic Sensing and Signal Processing for PD Detection in GIS[C]. Singapore:International Conference on Information, Communications and Signal Professing, 1997. 745 749.

[10] Bo Qi, Chengrong Li, Zhen Hao. The phenomenon and characteristics of the development of surface partial discharge of metal particles on the surface of GIS insulator[J]. Chinese Journal of Electrical Engineering, 2011,01:101 108.

[11] Ying Li, Wangdian Sun. Experimental signal processing based on MATLAB, Journal of Jinan University (NATURAL SCIENCE EDITION),2001,22(1).

[12] Zhixia Shi. Present situation and development of GIS field test [J]. High voltage electrical equipment , 2001, 37(4) :42-45. 